\title{
Imaging Amyloid in the Human Brain: A Promising Tool for Improved Early Diagnosis and Treatment Monitoring in Alzheimer's Disease
}

\author{
Alexander Drzezga ${ }^{1,2}$ and Robert Perneczky ${ }^{*}, 3,4$ \\ ${ }^{I}$ Harvard Medical School, Massachusetts General Hospital, Athinoula A. Martinos Center for Biomedical Imaging \\ Charlestown, MA, USA \\ ${ }^{2}$ Nuklearmedizinische Klinik, Klinikum rechts der Isar, Technische Universität München, Munich, Germany \\ ${ }^{3}$ Department of Clinical Neurosciences, University of Cambridge, School of Clinical Medicine, Cambridge, UK \\ ${ }^{4}$ Klinik und Poliklinik für Psychiatrie und Psychotherapie, Klinikum rechts der Isar, Technische Universität München, \\ Munich, Germany
}

\begin{abstract}
Alzheimer's disease has turned into one of today's most important medical and social challenges. Currently, the clinical diagnosis of Alzheimer's disease can only be established after the onset of the first symptoms. However, widespread and irreversible neural damage has probably already occurred at this stage. Furthermore, currently available drugs for Alzheimer's disease only offer a symptomatic treatment that can delay the progression of symptoms, with no effect on the neurodegeneration itself. Many drugs currently being evaluated for disease modifying effects in Alzheimer's disease will probably show their greatest effect in very early, pre-dementia disease stages, before widespread neural damage has occurred. Therefore, novel positron emission tomography tracers for amyloid, one of the histopathological hallmarks of Alzheimer's disease, are currently under evaluation regarding their abilities for pre-dementia diagnosis and treatment monitoring. First studies suggest that these tracers are able to reliably detect amyloid pathology in vivo, and that amyloid imaging is a promising candidate for the purpose of an improved early diagnosis and follow-up of Alzheimer's disease. This article discusses recent findings in this area and their relevance for the early diagnosis and treatment monitoring in Alzheimer's disease.
\end{abstract}

Keywords: Amyloid, imaging, PET, Alzheimer's disease, early diagnosis, treatment monitoring.

\section{INTRODUCTION}

Alzheimer's disease (AD) is a progressive neurodegenerative disorder that predominantly affects the elderly population. It can be classified into a sporadic form with a senile onset in most instances, which accounts for the majority of cases, and into a familial form with a pre-senile onset, in which causative gene mutations have been identified [1]. AD has turned into one of today's most important medical and social challenges, since it affects over 25 million individuals and their families worldwide [2], a number that is estimated to double until 2040. In addition to the individual suffering, the growing number of patients also has a great impact on the healthcare systems, causing immense costs. Although currently available medications such as cholinesterase inhibitors and memantine can delay the progression of cognitive symptoms, the effects are rather small and current drug treatment probably has no effect on neurodegeneration itself. Therefore, drugs that target the neurobiological substrates of cognitive deterioration are currently being developed.

*Address correspondence to this author at the (RP) Klinik und Poliklinik für Psychiatrie und Psychotherapie, or (AD) Nuklearmedizinische Klinik, Klinikum rechts der Isar, Technische Universität München, Ismaninger Str. 22, D-81675 München, Germany; Tel: (49)89-4140-4214(RP), -2971(AD); Fax: (49)89-4140-4888(RP), -4950 (AD);

E-mails: robert.perneczky@lrz.tum.de; a.drzezga@lrz.tum.de
The histopathological hallmarks of AD include intracellular neurofibrillary tangles, composed of hyperphosphorylated tau protein, and extracellular amyloid plaques throughout cortical and limbic brain regions. The amyloid deposits of insoluble protein material mainly contain high levels of the 40 and 42 amino acid long amyloid $\beta$ peptides $(A \beta) . A \beta$ is produced through the cleavage of the amyloid precursor protein (APP) by the $\beta$ - and $\gamma$-secretases. The accumulation of $A \beta$ (in particular of $A \beta 42$ ) in the brain initiates a cascade of events, which eventually leads to neuronal dysfunction, neurodegeneration, and dementia [3]. The histopathological proof of abundant amyloid-plaque and neurofibrillary pathology is essential for the diagnosis of definite AD. Although the exact mechanisms are not entirely understood, amyloid-aggregation is widely accepted to play a major role in the pathogenesis of $\mathrm{AD}$. As a consequence, therapeutic strategies that target the accumulation and aggregation of amyloid are gaining importance. Promising candidates for a successful causal treatment strategy include immunization against $A \beta$ and the reduction of enzyme, i.e. secretase, activity that is crucial for its production [4].

Many of the drugs being currently evaluated will probably show their greatest effects in early disease stages with absent or only mild clinical symptoms. It is commonly assumed that neuronal damage, once it has occurred in the brain, is largely irreversible. Therapy approaches therefore can be expected to be more successful when they are initiated in an early disease stage before widespread 
irreversible neuronal damage has occurred. This may also be the reason why results from the first $A \beta$-immunization study in patients with manifest $\mathrm{AD}$ suggest that the removal of amyloid plaques is not necessarily associated with cognitive improvement $[5,6]$. However, the reliable diagnosis of AD in earlier stages is limited by a number of considerations. First, the clinical classification of $\mathrm{AD}$ based on neuropsychological tests has a rather low sensitivity of $80 \%$ and specificity of $70 \%$ even in cases of manifest dementia [7]. Second, it is commonly accepted that AD-associated neurodegeneration starts many years before the first clinical symptoms of dementia occur; therefore, the disease is already in an advanced stage if clinical criteria for dementia are met [8]. Consequently, the success of causal treatment approaches may depend on initiation in pre-dementia stages. In fact, today many therapy trials are aiming at the group of patients with mild cognitive impairment (MCI) with first cognitive symptoms that are not of sufficient magnitude to warrant the diagnosis of dementia [9]. In this pre-dementia stage patients have an increased risk to develop $\mathrm{AD}$ with rates of progression to dementia up to $15 \%$ per year [10]. However, there are also patients who fulfil the diagnostic criteria for MCI but will not develop dementia for a long time or even revert to normal. So far, the reliable prediction of dementia in MCI cannot be achieved using clinical and neuropsychological measures alone. Finally, the differentiation between $\mathrm{AD}$ and other dementia syndromes such as frontotemporal dementia and dementia with Lewy bodies is often limited by the significant overlap of symptoms [7].

Not only the diagnosis but also the monitoring of treatment effects is sometimes difficult using neuropsychological evaluations alone due to high rates of inter- and intraindividual variations and small clinical treatment effects. Comparable levels of cognitive impairment between different subjects at baseline may not warrant comparable levels of pathology in the brain. Furthermore, symptomatic changes may not always directly mirror cerebral changes. Inter-individual differences in the degree of cognitive reserve, which probably acts as a counterweight against brain damage, may further contribute to a dissociation between the extent of pathology and the degree of measurable cognitive impairment [11].

Reliable biomarkers that measure brain changes related to $\mathrm{AD}$ pathology are therefore urgently needed to improve early diagnosis and treatment monitoring in the advent of novel causal treatment options.

\section{IMAGING OF CEREBRAL AMYLOID DEPOSITION}

The development of in vivo tracers of amyloid pathology, using positron emission tomography (PET) to visualize cerebral amyloid depositions, provides an intriguing tool to study AD pathology ante mortem. Using this technique of 'in vivo histopathology' allows to study neurodegenerative disorders on the basis of their neuropathological underpinning rather than their clinical symptoms. Several tracers have been developed so far. Among these tracers, [11C]PIB (Pittsburgh Compound B) [12] is the best established, and several F-18 labelled tracers (with a longer half-life, suitable for wider distribution) are currently under evaluation in large clinical studies. Examples are [18F]Florbetaben by Bayer-Schering Pharma AG, [18F]AV-
45 by AVID Radiopharmaceuticals and [18F]PIB by GE Healthcare. By now, a number of studies addressed the value of amyloid-plaque imaging tracers. In vitro studies, comparing histological staining with autoradiography studies on tissue samples of patients with $\mathrm{AD}$ were able to demonstrate that [11C]PIB seems to be specific for amyloid aggregates and shows only neglectable binding to other protein aggregates such as neurofibrillary tangles, Lewy bodies or ubiquitin [13]. This does of course have important implications for therapy studies directed against a specific type of protein aggregation pathology. While being specific for amyloid pathology in general, [11C]PIB apparently binds to different variants of amyloid-aggregation pathology and, thus, neither allows for a differentiation between different types of plaques nor between plaques and cerebral amyloid angiopathy [13]. Another tracer for amyloid-imaging, [18F]FDDNP, has been discussed to show affinity not only to plaques but also to neurofibrillary tangles [14]. In recent studies, however, this assumption has been questioned and a low overall affinity of this tracer to amyloid-plaques as well as to neurofibrillary tangles has been demonstrated [15].

Apart from in vitro experiments, a number of studies indicate that this new generation of tracers does in fact allow visualization of amyloid plaques in vivo. It has been consistently shown that in comparison with healthy control subjects, patients with $\mathrm{AD}$ have significantly higher [11C]PIB tracer-uptake in brain regions which are expected to be amyloid-rich [16]. First studies also indicate that the tracer-uptake corresponds to histopathologically confirmed amyloid depositions [17]. This will however have to be confirmed in larger systematically acquired samples. Differences between the amyloid-tracer-uptake, with peak levels in the frontal cortex, and the pathological findings in brain functional studies, rather located in more posterior brain regions [18], suggest that amyloid pathology may not be directly responsible for neuronal dysfunction in $\mathrm{AD}$.

With regard to the specificity of the tracer, studies with [11C]PIB-PET reported no increased tracer-uptake in most patients with frontotemporal lobar degenerations, in which amyloid pathology is not present [19-21]. These results strongly indicate a role of this tracer for differential diagnosis between different neurodegenerative disorders on the basis of the underlying pathology rather than on the symptomatic appearance. On the other hand, in dementia with Lewy bodies, in which amyloid pathology is frequently found in addition to alpha-synuclein aggregates, [11C]PIBPET tracer-uptake can be similar to AD [21], limiting the differential diagnostic value of amyloid imaging in this context. However, the tracer may allow for the identification of plaque-positive and plaque-negative patients with dementia with Lewy bodies, which may have implications for prognosis and therapy. Regarding the value of amyloidimaging for the early diagnosis of AD-pathology in predementia stages, positive [11C]PIB-PET scans were associated with an increased risk for dementia in MCI [22]. Cortical [11C]PIB tracer-uptake is also found in almost a third of cognitively healthy elderly subjects [16]. This corresponds to the repeated observation that up to $30 \%$ of healthy individuals over the age of 75 show $\mathrm{A} \beta$ deposition at autopsy $[23,24]$. These individuals may suffer from preclinical $\mathrm{AD}$, an assumption that seems to be strengthened by first amyloid imaging follow-up studies [25, 26]. However, 
further studies will have to clarify the relationship between amyloid pathology and the risk for AD in clinically healthy persons. Furthermore, a cogent explanation of the nonspecific white matter tracer binding regardless of the diagnosis is still missing.

\section{LONGITUDINAL STUDIES AND THE MONITORING OF TREATMENT EFFECTS}

For an optimal disease progression marker there should be a linear relationship between the degree of actual neuropathology and the marker signal; in other words, the marker should be able to reliably quantify the neuropathological changes. So far, this linear relationship between the extent of pathology and the tracer-uptake could not be sufficiently established for the available amyloidPET-tracers and further in vivo/ex vivo studies are required for that purpose. Nevertheless, it can be assumed that higher tracer uptake represents higher amyloid-plaque load in the brain. This may be important for the definition of a reliable baseline in therapy studies, particularly with regard to the potentially weak association between cognitive impairment and the extent of cerebral pathology. In fact, first studies were already able to demonstrate that comparable levels of cognitive impairment may be present in spite of differences in amyloid-plaque pathology in the brain. One study demonstrated higher amyloid-plaque levels in subjects with higher education in spite of comparable cognitive decline as in less educated subjects, pointing to a cognitive reserve effect [27]. Another study demonstrated a higher amyloidplaque load in subjects positive for the $A P O E$ e4-allele (the strongest genetic risk factor for AD), in spite of comparable age and disease duration as in $A P O E$ e4-negative subjects, pointing to an accelerated amyloid-deposition in e4-positives [28]. These studies underline that the inclusion of subjects in therapy trials on the basis of clinical variables may not warrant comparable levels of baseline pathology and potentially distort treatment effects. Amyloid-plaque imaging may serve as a more objective measure for the quantification of baseline pathology and of therapy effects.

It is not yet known if changes in amyloid pathology will

\section{Amyloid-plaque imaging of potential therapy effects in Alzheimer's disease}

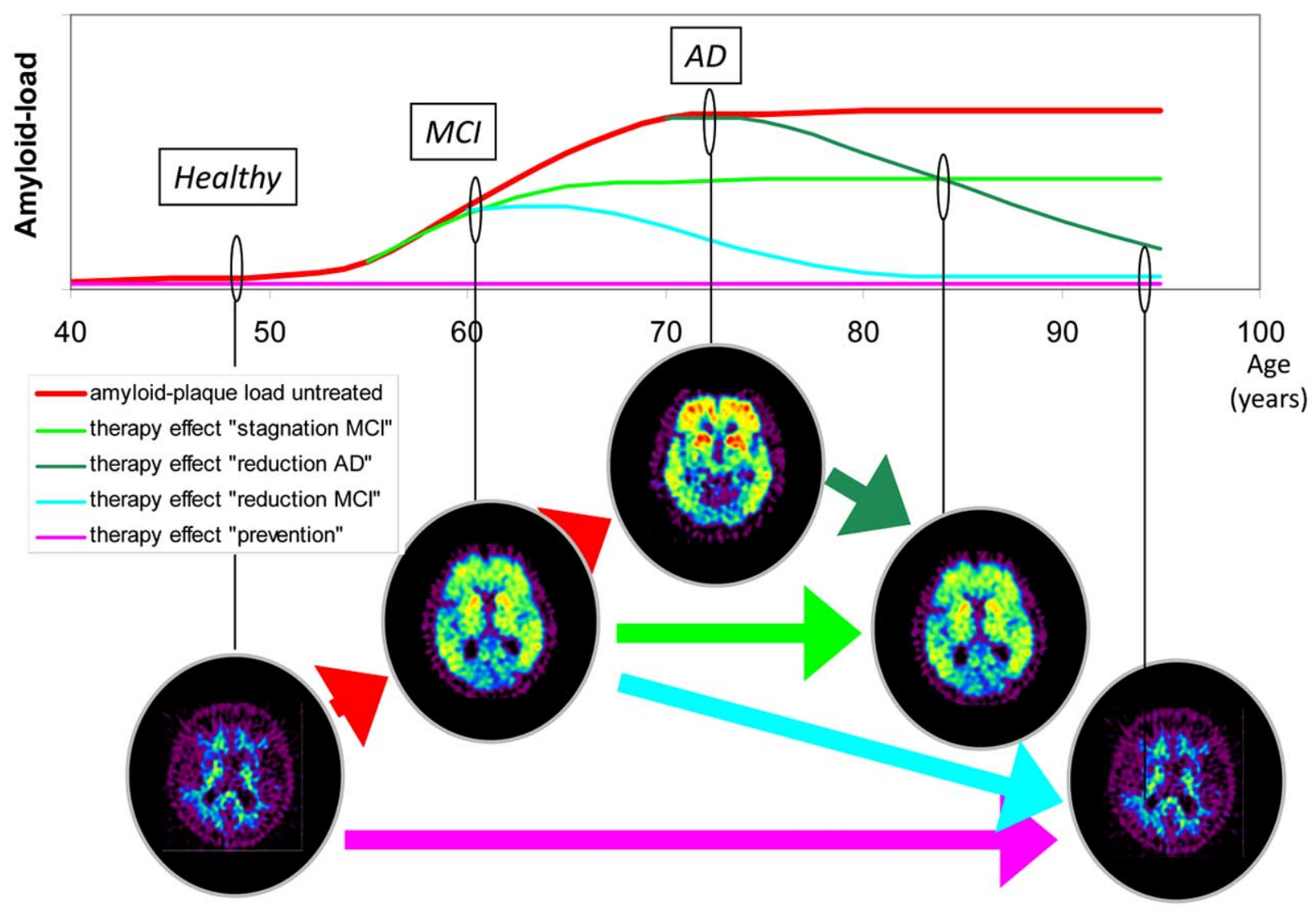

Fig. (1). Potential applications of amyloid-plaque imaging for measuring treatment effects of anti-amyloid therapy approaches in Alzheimer's disease. Diagram: Red line: Assumed natural course of amyloid-plaque deposition in the brain of patients suffering from Alzheimer's disease. Other lines: treatment-induced modification of the natural course, depending on time point of treatment initiation and treatment effect (reducing existing amyloid-plaque load or preventing further aggregation). PET-images: [11C]PIB scans, colour-code: red represents high tracer-uptake indicating high amyoid-plaque load, yellow: intermediate tracer-uptake, blue: low tracer uptake. AD: Alzheimer's disease, MCI: Mild cognitive impairment (Figure reproduced in modified form with friendly permission by Drzezga A, DementiaEurope 2009 [35]). 
be mirrored by changes in tracer-uptake. First longitudinal studies showed that [11C]PIB uptake remained stable within one year in an $\mathrm{AD}$ group, whereas glucose metabolism measured with [18F]FDG PET decreased within the same period [29]. This finding is possibly explained by the fact that amyloid deposition may have already reached a plateau by the time when the threshold to dementia is crossed. Neuronal damage mirrored by metabolism in [18F]FDG PET on the other hand still advances at the same time. This assumption may also explain why cognitive performance and [11C]PIB uptake do not seem to be tightly linked $[16,30]$. Furthermore, these results support the notion that [18F]FDG PET will continue to play an important role in the diagnostic assessment and follow-up of dementia, particularly with regard to treatment effects.

At present, it is still a matter of debate whether (1) antiamyloid therapies can actually reduce or stop amyloid deposition, and if (2) such a reduction can be monitored using amyloid imaging. In a first attempt, treatment with phenserine, a cholinesterase inhibitor with some amyloidlowering properties has been followed in a group of $\mathrm{AD}$ patients with [11C]PIB PET over three to six months, but no systematic reduction of [11C]PIB uptake was found [31]. Major limitations of amyloid imaging can be found in the still limited number of in vivo/ex vivo studies documenting that amyloid tracer uptake indeed corresponds to amyloidplaque load in the brain. In particular, translation of tracer signal into quantity of actual pathology in the brain needs to be established. Finally, it is currently discussed that soluble stages of amyloid-aggregation (oligomeres) may represent the main causal pathology in the genesis of $\mathrm{AD}$ rather than the insoluble amyloid plaques [32]. This may explain why the number of aggregated amyloid-plaques may not necessarily show a tight correlation to cognitive performance and it may also limit the utility of amyloid-plaque imaging for treatment effects directed against the production of oligomeres rather than towards elimination of amyloidplaques (Fig. 1).

Further studies, including histopathological verification of diagnoses, longer follow-up periods (particularly in amyloid-positive healthy subjects) and suitable small animal experiments will be required to further elucidate the usefulness of amyloid imaging for diagnostic purposes and for treatment monitoring in AD. Several new amyloid tracers are under evaluation using animal models of $\mathrm{AD}$ that could yield higher signal-to-noise-ratios resulting in an improved sensitivity and specificity in human studies. Johnson et al. for example recently reported preclinical results on the [11C] labelled tracer AZD2184, which suggest a low non-specific background binding compared with the reference ligand PIB [33]. However, it must be pointed out that findings in transgenic animals may depend on the type of animal model used and are not always reflected in patients with $\mathrm{AD}$, possibly due to differences in the time course of amyloid deposition or tissue factors present during deposition that lead to an alternative secondary structure of amyloid [34].

\section{CONCLUSION}

Anti-amyloid therapies and reliable biomarkers of disease severity and progression in $\mathrm{AD}$ will have to be developed simultaneously to exploit their full potential.
Amyloid imaging is a promising candidate for the purpose of an improved early diagnosis and follow-up of AD. It will possibly help to improve the identification of individuals at risk for dementia at very early stages without advanced neurodegeneration, and therefore help to prevent further irreversible brain damage. Amyloid imaging may also help to prevent possible harmful treatment side effects in patients with non-AD neurodegeneration that mimics $\mathrm{AD}$ symptoms. In terms of treatment monitoring, even the documentation of a stagnating tracer uptake in pre-dementia stages may be regarded as proof of successful anti-amyloid treatment. However, a reliable definition of treatment success will have to be established first for anti-amyloid drugs.

To conclude, imaging amyloid in the human brain is a promising tool for improved early diagnosis and treatment monitoring in $\mathrm{AD}$, if the remaining open questions on its properties can be resolved.

\section{ACKNOWLEDGEMENTS}

This work has been supported in part by DFG-grants (Deutsche Forschungsgemeinschaft) Project Numbers: DR 445/3-1, DR 445/4-1 (Drzezga), and a grant from the German Society for Psychiatry, Psychotherapy and Nervous Diseases (Deutsche Gesellschaft für Psychiatrie, Psychotherapie und Nervenheilkunde; Perneczky).

\section{REFERENCES}

[1] Blennow K, de Leon MJ, Zetterberg H. Alzheimer's disease. Lancet 2006; 368: 387-403.

[2] Wimo A, Winblad B, Aguero-Torres H, et al. The magnitude of dementia occurrence in the world. Alzheimer Dis Assoc Disord 2003; 17: 63-7.

[3] Hardy J, Selkoe DJ. The amyloid hypothesis of Alzheimer's disease: progress and problems on the road to therapeutics. Science 2002; 297: 353-6.

[4] Hull M, Berger M, Heneka M. Disease-modifying therapies in Alzheimer's disease: how far have we come? Drugs 2006; 66: 2075-93.

[5] Hock C, Konietzko U, Streffer JR, et al. Antibodies against betaamyloid slow cognitive decline in Alzheimer's disease. Neuron 2003; 38: 547-54.

[6] Gilman S, Koller M, Black RS, et al. Clinical effects of Abeta immunization (AN1792) in patients with $\mathrm{AD}$ in an interrupted trial. Neurology 2005; 64: 1553-62.

[7] Knopman DS, DeKosky ST, Cummings JL, et al. Practice parameter: diagnosis of dementia (an evidence-based review). Report of the Quality Standards Subcommittee of the American Academy of Neurology. Neurology 2001; 56: 1143-53.

[8] Braak E, Griffing K, Arai K, et al. Neuropathology of Alzheimer's disease: what is new since A. Alzheimer? Eur Arch Psychiatry Clin Neurosci 1999; 249 (Suppl 3): 14-22.

[9] Petersen RC. Mild cognitive impairment as a diagnostic entity. J Intern Med 2004; 256: 183-94.

[10] Boyle PA, Wilson RS, Aggarwal NT, et al. Mild cognitive impairment: risk of Alzheimer disease and rate of cognitive decline. Neurology 2006; 67: 441-5.

[11] Perneczky R, Drzezga A, Diehl-Schmid J, et al. Schooling mediates brain reserve in Alzheimer's disease: findings of fluorodeoxy-glucose-positron emission tomography. J Neurol Neurosurg Psychiatry 2006; 77: 1060-3.

[12] Klunk WE, Engler H, Nordberg A, et al. Imaging brain amyloid in Alzheimer's disease with Pittsburgh Compound-B. Ann Neurol 2004; 55: 306-19.

[13] Lockhart A, Lamb JR, Osredkar T, et al. PIB is a non-specific imaging marker of amyloid-beta (Abeta) peptide-related cerebral amyloidosis. Brain 2007; 130: 2607-15.

[14] Small GW, Kepe V, Ercoli LM, et al. PET of brain amyloid and tau in mild cognitive impairment. N Engl J Med 2006; 355: 2652-63. 
[15] Thompson PW, Ye L, Morgenstern JL, et al. Interaction of the amyloid imaging tracer FDDNP with hallmark Alzheimer's disease pathologies. J Neurochem 2009; 109: 623-30.

[16] Pike KE, Savage G, Villemagne VL, et al. Beta-amyloid imaging and memory in non-demented individuals: evidence for preclinical Alzheimer's disease. Brain 2007; 130: 2837-44.

[17] Ikonomovic MD, Klunk WE, Abrahamson EE, et al. Post-mortem correlates of in vivo PiB-PET amyloid imaging in a typical case of Alzheimer's disease. Brain 2008; 131: 1630-45.

[18] Li Y, Rinne J, Mosconi L, et al. Regional analysis of FDG and PIB-PET images in normal aging, mild cognitive impairment, and Alzheimer's disease. Eur J Nucl Med Mol Imaging 2008; 35: 2169 81.

[19] Drzezga A, Grimmer T, Henriksen G, et al. Imaging of amyloid plaques and cerebral glucose metabolism in semantic dementia and Alzheimer's disease. Neuroimage 2008; 39: 619-33.

[20] Engler H, Santillo AF, Wang SX, et al. In vivo amyloid imaging with PET in frontotemporal dementia. Eur J Nucl Med Mol Imaging 2008; 35; 100-6.

[21] Rowe $\mathrm{CC}, \mathrm{Ng} \mathrm{S}$, Ackermann U, et al. Imaging beta-amyloid burden in aging and dementia. Neurology 2007; 68: 1718-25.

[22] Okello A, Koivunen J, Edison P, et al. Conversion of amyloid positive and negative MCI to AD over 3 years. An 11C-PIB PET study. Neurology 2009; 73; 745-60.

[23] Jorm A, Jolley D. The incidence of dementia: a meta-analysis. Neurology 1989; 51: 728-33.

[24] Riley KP, Snowdon DA, Markesbery WR. Alzheimer's neurofibrillary pathology and the spectrum of cognitive function: findings from the Nun Study. Ann Neurol 2002; 51: 567-77.

[25] Villemagne V, Pike K, Darby D, et al. A $\beta$ deposits in older nondemented individuals with cognitive decline are indicative of preclinical Alzheimer's disease. Neuropsychologia 2008; 46: 168897.
[26] Scheinin N, Aaalto S, Koikkalainen J, et al. Follow-up of [11C]PIB uptake and brain volume in patients with Alzheimer disease and controls. Neurology 2009; 73; 1186-92.

[27] Kemppainen NM, Aalto S, Karrasch M, et al. Cognitive reserve hypothesis: Pittsburgh Compound B and fluorodeoxyglucose positron emission tomography in relation to education in mild Alzheimer's disease. Ann Neurol 2008; 63: 112-8.

[28] Drzezga A, Grimmer T, Henriksen G, et al. Effect of APOE genotype on amyloid plaque load and gray matter volume in Alzheimer disease. Neurology 2009; 72: 1487-94.

[29] Engler H, Forsberg A, Almkvist O, et al. Two-year follow-up of amyloid deposition in patients with Alzheimer's disease. Brain 2006; 129: 2856-66.

[30] Grimmer T, Henriksen G, Wester HJ, et al. Clinical severity of Alzheimer's disease is associated with PIB uptake in PET. Neurobiol Aging 2009; 30; 1902-9.

[31] Kadir A, Andreasen N, Almkvist O, et al. Effect of phenserine treatment on brain functional activity and amyloid in Alzheimer's disease. Ann Neurol 2008; 63: 621-31.

[32] Selkoe DJ. Soluble oligomers of the amyloid beta-protein impair synaptic plasticity and behavior. Behav Brain Res 2008; 192: 10613.

[33] Johnson A, Jeppsson F, Sandell J, et al. AZD2184: a radioligand for sensitive detection of beta-amyloid deposits. J Neurochem 2009; 108: 1177-86.

[34] Klunk W, Lopresti B, Ikonomovic M, et al. Binding of the positron emission tomography tracer Pittsburgh compund-B reflects the amount of amyloid-beta Alzheimer's disease brain but not in transgenic mouse brain. J Neurosci 2005; 25: 10598-606.

[35] Drzezga A. Amyloid-plaque imaging: a tool to study Alzheimer's disease modification? Dement Eur 2009; 1: 6-7.

(C) Drzezga and Perneczky; Licensee Bentham Open.

This is an open access article licensed under the terms of the Creative Commons Attribution Non-Commercial License (http: //creativecommons.org/licenses/by-nc/ 3.0/) which permits unrestricted, non-commercial use, distribution and reproduction in any medium, provided the work is properly cited. 